\title{
A Hypoelastic Dynamic Constitutive Model to Account for the Hysteretic Behaviour of Soil Subjected to Cyclic Loads
}

\author{
Jian Li, ${ }^{1}$ Jianhua Guo $\mathbb{D}^{1,},{ }^{1,2}$ Zhangjun Dai $\mathbb{D}^{1},{ }^{1}$ Lingfa Jiang, ${ }^{1}$ and Shanxiong Chen ${ }^{1}$ \\ ${ }^{1}$ State Key Laboratory of Geomechanics and Geotechnical Engineering, Institute of Rock and Soil Mechanics, \\ Chinese Academy of Sciences, Wuhan 430071, China \\ ${ }^{2}$ University of Chinese Academy of Sciences, Beijing 100049, China \\ Correspondence should be addressed to Jianhua Guo; guojianhua17@mails.ucas.edu.cn
}

Received 23 July 2020; Revised 5 November 2020; Accepted 20 February 2021; Published 28 February 2021

Academic Editor: Xue Zhang

Copyright (c) 2021 Jian Li et al. This is an open access article distributed under the Creative Commons Attribution License, which permits unrestricted use, distribution, and reproduction in any medium, provided the original work is properly cited.

\begin{abstract}
To reduce the difficulties associated with dynamic constitutive models, a model was established for soil in this study based on hypoelasticity. The stress-strain relationship in soil under a cyclic load was divided into three stages: initial loading, unloading, and reloading. The stress-strain relationship in each stage was ascertained using a hyperbolic equation. On this basis, the physical significance of the parameters in the model and their method of determination were described. The effects of the parameters on the stress-strain relationship were investigated and the integration algorithm of the model was established. Finally, the rationality of the proposed model was verified by conducting triaxial tests under conventional and cyclic loads. The results show that the model is able to adequately demonstrate all the stress-strain relations in the soil under both static and dynamic loads.
\end{abstract}

\section{Introduction}

Dynamic constitutive models are important tools to describe the dynamic characteristics of soil under cyclic loads [1-3]. The main difference between a conventional static constitutive model and a dynamic one is that the latter can accurately delineate the hysteretic behaviour of the soil under dynamic loads $[4,5]$. At present, the existing constitutive models used in the geotechnical field to reveal the dynamics of soil under cyclic loads can be divided into three main types [6, 7]: elastoplastic and viscoplastic dynamic constitutive models and transient modulus field models (based on transient-limit equilibrium theory).

There is an abundant variety of dynamic constitutive models based on elastoplastic theory currently in use in research. The models were first developed from the multiyield-surface model established by Mroz et al. [8, 9]. In them, a series of yield surfaces are applied to simulate the nonlinear and hysteretic nature of the dynamic stress-strain behaviour of the soil. Moreover, these yield surfaces constantly move within the stress space as the stress state of the soil changes. By setting a rule to demonstrate the movement of the yield surfaces, the stress history of the soil can be recorded to exhibit the nonlinearity of the dynamic stress-strain relationship within the soil. There are a variety of improved multiyield-surface models and numerous scholars [10-12] have established their own multiyield-surface models. Several authors in China [13-17] have also proposed specific models.

Multiyield-surface models are based on complicated theory and tedious rules. Although this means they facilitate a flexible description of the dynamic stress-strain variation in the soil, it creates drawbacks as well. For example, a lengthy calculation process is required during the development and application of such models due to the excessively complex theory and tedious rules required. In particular, the models are rather poor in practical applications where large-scale numerical calculations are required. Dafalias et al. [18-22] built a boundary surface model with a single face to overcome this drawback. In this model, all the small yield surfaces within the maximum yield surface are removed and replaced by mapping rules. As a result, the difficulties encountered in the development and application of multiyield-surface models could be reduced, so they could 
be further developed in subsequent research. The proposed plasticity constitutive model of anisotropic clay is more concise and does not change the structure of the original equation.

The transient modulus field model was first proposed by Xie et al. [23-25]. It was used to analyse the constitutive relationship in saturated sand and is based on transient-limit equilibrium theory. In the model, the stress-strain behaviour is divided into three stages. A transient modulus field is established in each stage to reflect the nonlinearity and hysteretic nature of the stress-strain relationship. Such models have a short development time and are mainly used to study saturated sand. Therefore, their applicability to other soils, for example, clay, warrants further verification.

The endochronic model first proposed by Valanis was used to describe the nonlinearity of materials based on viscoplastic behaviour. Bazant et al. [26-28] and Zienkiewicz et al. [29] later utilized the model to describe various aspects of soil under cyclic loads (including dynamic stress-strain relationship, plastic deformation, and increase in pore pressure). Endochronic models depict the internal changes in materials via the endochronic parameter $Z$. This parameter is used to reflect material properties and employs a monotonically increasing function of time. The conversion variables of the endochronic parameter are used to delineate various irrecoverable linear properties of rock and soil materials during stress processes including cumulative plastic strain and pore pressure. As endochronic theory does not consider the complex concept of yield function (which does feature in elastoplastic mechanics theory), it has a simpler theoretical form than elastoplastic theory. However, endochronic theory involves semiempirical test functions whose parameters do not have obvious physical significance. This makes the parameters difficult to determine so that the theory has limited practical applicability.

To sum up, the following problems associated with dynamic constitutive models need to be solved in subsequent research: (i) the models need to accurately reflect the real stress-strain behaviour of the soil, especially its hysteretic nature. (ii) They should have a simple theoretical form and thus avoid development difficulties because of complex and tedious rules. (iii) The models' parameters need to be as simple as possible and have specific physical significance. They should also be convenient to acquire (via tests if needed), so the model is easy to apply to practical applications.

From the introduction above, it can be found that, among the various types of dynamic constitutive model, the bounding surface model was the most popular on account of its relatively simple form, but it should be noted that the bounding surface model also lies within the framework of elastoplastic theory, and, in its application, many partial differential equations must be solved. Therefore, it still seems to be a too complicated model to use. In this study, a new simple dynamic constitutive model based on the theory of hypoelasticity and hyperbolic equations has been established. The original purpose of its proposal is to simplify the application of the dynamic constitutive model. In the model, the stress-strain relationship of the soil under cyclic loads is divided into three stages. A hyperbolic equation is adopted to reveal the stress-strain behaviour of the soil in each stage. In addition, the strain in the soil corresponding to the current stress state is calculated using an incremental integration method, thus avoiding complex elastoplastic calculations. As a result, the model has a simple form and can be conveniently applied in large-scale numerical calculations.

\section{Construction of the Model}

In 1963, Kondner pointed out that the curves obtained from triaxial tests conducted on soil can be accurately fitted to equations of hyperbolic form. This observation was based on a large number of stress-strain curves obtained through conventional triaxial tests. That is, the stress $(\sigma)$ and strain $\left(\varepsilon_{a}\right)$ appear to obey a relationship of the form

$$
\sigma_{1}-\sigma_{3}=\frac{\varepsilon_{a}}{a+b \varepsilon_{a}}
$$

where $a$ and $b$ are constants.

On this basis, Duncan and Chang [30] established an incremental hypoelasticity model based on stress-strain curves which is widely used at present (the "DuncanChang" model). The stress-strain behaviour of soil under monotonic loading can be well described using the Duncan-Chang model. In light of the success of the DuncanChang model, the stress-strain behaviour of soil under cyclic loading is investigated here using that model for inspiration.

Figure 1 illustrates the typical dynamic stress-strain behaviour exhibited by the soil under a cyclic load. The stress-strain curve exhibits obvious signs of hysteresis under the cyclic load. If unloading and reloading are considered as the cyclic loading period, then the stress-strain variation in each cyclic period essentially has the same characteristics. Thus, the cyclic loading and unloading procedure can be divided into several stages and the stress-strain relationship within each loading stage is described using hyperbolic curves. The stress-strain relationship of the soil is fitted using a treatment based on incremental hypoelasticity theory. Thus, the application of hypoelasticity theory can be extended to the fitting of the stress-strain behaviour of the soil under cyclic loads.

Figure 1 illustrates a typical stress-strain curve for the soil under a cyclic load. It can be seen that the stress-strain variation can be divided into two stages: unloading and reloading. Moreover, combining this with the initial loading stage, the stress-strain relationship of the soil during the complete process can be partitioned into three stages: initial loading, unloading, and reloading. Of course, under cyclic loads, the unloading and reloading stages occur cyclically. In addition, the unloading and reloading curves in each load period can be fitted to a hyperbolic expression.

2.1. Initial Loading Stage. Clearly, the stress-strain curve of the soil in the initial loading stage can be well described using a hyperbolic curve; that is, 


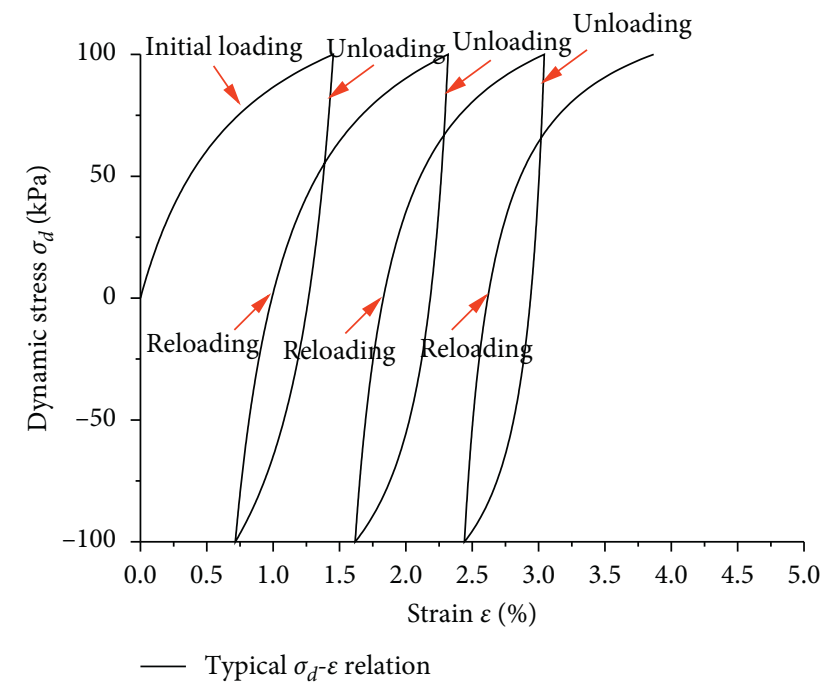

FIgURE 1: Typical stress-strain behaviour under a cyclic load.

$$
\begin{gathered}
\sigma=\frac{\varepsilon_{1}}{a_{i}+b_{i} \varepsilon_{1}}, \\
\sigma=\sigma_{1}-\sigma_{3},
\end{gathered}
$$

where $a_{i}$ and $b_{i}$ are the model parameters for the initial loading stage (Figure 2).

2.2. Unloading Stage. The stress-strain relationship of the soil in the unloading stage during each load period is regarded as a separate curve. The stress-strain curve during a particular unloading stage is considered as the curve obtained by translating a hyperbolic curve identical to the original along the coordinate axis. The relationship is thus expressed as follows:

$$
\begin{aligned}
\sigma-\sigma_{0} & =\frac{\left(\varepsilon_{1}-\varepsilon_{0}\right)}{a_{u}-b_{u}\left(\varepsilon_{1}-\varepsilon_{0}\right)} \\
\sigma & =\sigma_{1}-\sigma_{3}, \quad\left(\sigma_{0}>0, \varepsilon_{0}>0\right),
\end{aligned}
$$

where $a_{u}$ and $b_{u}$ represent the model parameters in the unloading stage. The quantities $\sigma_{0}$ and $\varepsilon_{0}$ refer to the stress translation and strain translation, respectively (Figure 3 ).

2.3. Reloading Stage. In a similar manner, the stress-strain curve of the soil in the reloading stage in each load period is considered to be a separate curve. Therefore, the stress-strain curve in the reloading stage can also be regarded as a translated hyperbolic curve. Thus,

$$
\begin{aligned}
\sigma-\sigma_{0} & =\frac{\left(\varepsilon_{1}-\varepsilon_{0}\right)}{a_{r}+b_{r}\left(\varepsilon_{1}-\varepsilon_{0}\right)} \\
\sigma & =\sigma_{1}-\sigma_{3}, \quad\left(\sigma_{0}<0, \varepsilon_{0}>0\right),
\end{aligned}
$$

where $a_{r}$ and $b_{r}$ denote the model parameters in the reloading stage. Again, the parameters $\sigma_{0}$ and $\varepsilon_{0}$ represent

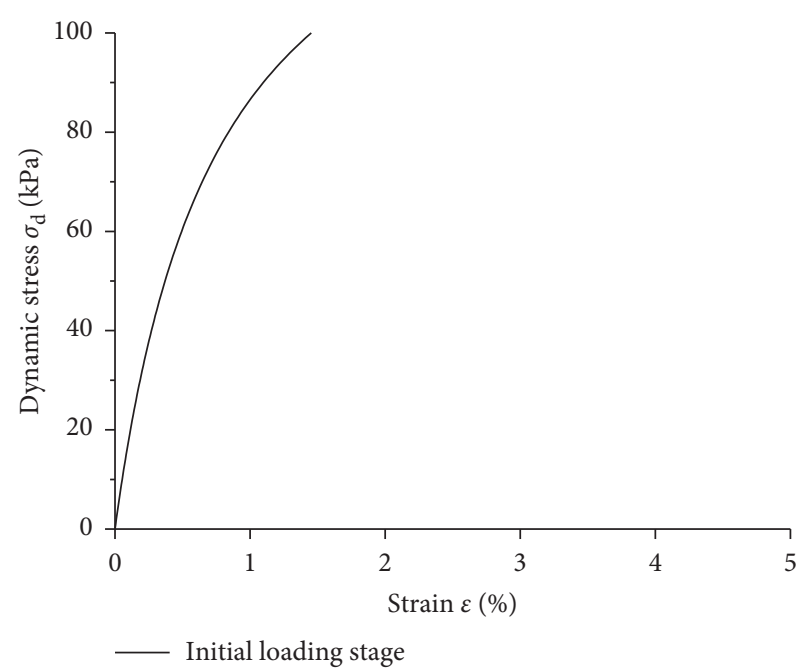

Figure 2: A typical stress-strain curve for the initial loading stage.

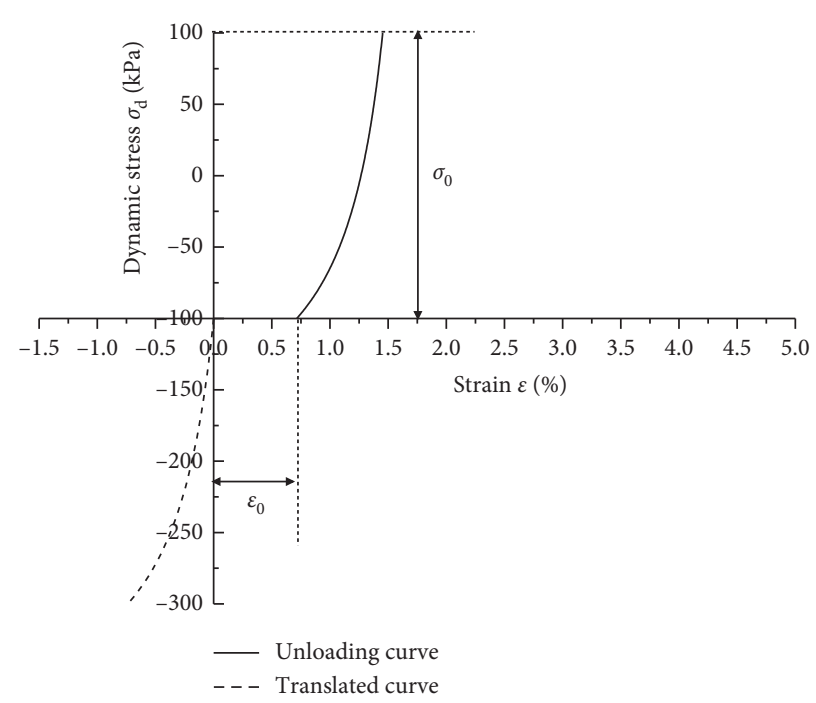

Figure 3: Stress-strain and translated stress-strain curves in the unloading stage.

the stress translation and strain translation, respectively (Figure 4).

\section{Significance and Method of Determination of the Model Parameters}

For the model constructed in this study, two crucial parameters, that is, $a$ and $b$, which control the basic pattern of the hyperbolic curves, are determined. As mentioned above, in order to describe the hysteresis in the stress-strain curve under a cyclic load, the stress deformation of the soil is divided into three stages. In each stage, the stress is fitted using a hyperbolic equation. This means that there are six test parameters in the model $\left(a_{i}\right.$ and $b_{i}$ in the initial loading stage, $a_{u}$ and $b_{u}$ in the unloading stage, and $a_{r}$ and $b_{r}$ in the reloading stage). 


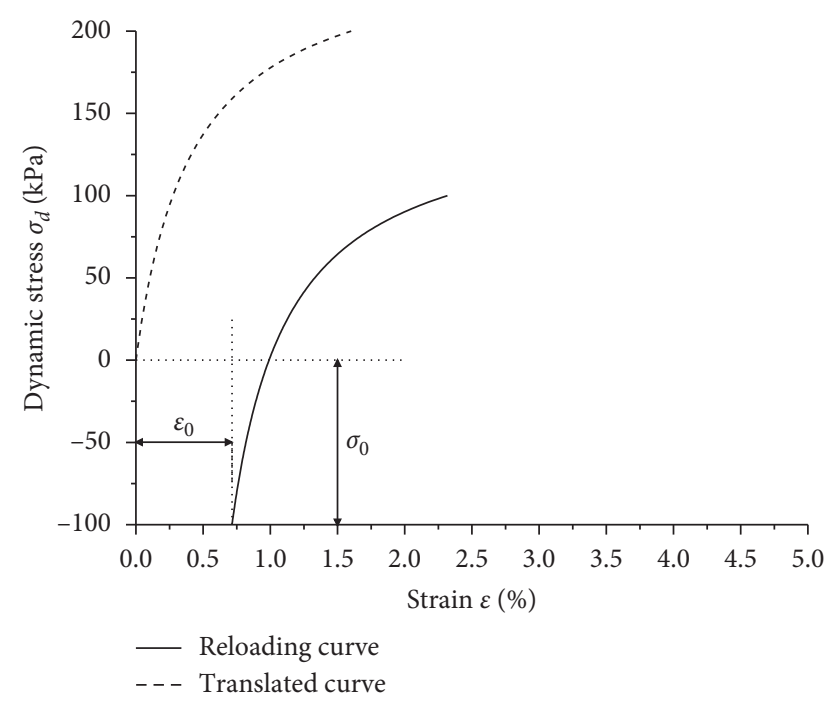

FIGURE 4: Stress-strain and translated stress-strain curves in the reloading stage.

\subsection{Parameter a}

3.1.1. Initial Loading Stage. It can be shown using equation (2) that, during the initial loading stage, the tangent modulus of the stress-strain curve is expressed using the differential of the stress to strain as follows:

$$
\frac{\mathrm{d} \sigma}{\mathrm{d} \varepsilon_{1}}=\frac{a_{i}}{\left(a_{i}+b_{i} \varepsilon_{1}\right)^{2}}
$$

As $\varepsilon_{1} \longrightarrow 0$, the initial tangent modulus of the stressstrain curve is, therefore, $E_{t i}=1 / a_{i}$. That is to say, the parameter $a_{i}$ in the initial loading stage is equal to the initial tangent modulus of the curve, that is, $a_{i}=1 / E_{t i}$ (Figure 5).

3.1.2. Unloading Stage. In a similar way, the parameter $a_{u}$ of the stress-strain curve in the unloading stage is calculated through the appropriate gradient. That is, $a_{u}=1 / E_{t u}$ (Figure 6). However, it is worth noting that stresses and strains decline under unloading conditions, so the initial tangent modulus $E_{t u}$ of the curve is negative.

3.1.3. Reloading Stage. Once again, the parameter $a_{r}$ in the reloading stage can be determined to be reciprocal of the initial tangent modulus of the reloading curve; that is, $a_{r}=$ $1 / E_{t r}$ (Figure 7 , where $E_{r u}$ is seen to have a positive value).

3.2. Parameter $b$. Unlike the Duncan-Chang model, the parameter $b$ in the model constructed here does not have a definite physical significance. However, its value can be readily calculated from the test data by taking derivatives.

The stress-strain relationship in the soil is partitioned into three stages in the model. In each loading stage, the origin $\left(\sigma_{0}, \varepsilon_{0}\right)$ and end $\left(\sigma_{1}, \varepsilon_{1}\right)$ of the stress-strain curve are defined, as shown in Figures 5-7. Moreover, the secant moduli in each loading stage (initial loading, unloading, and

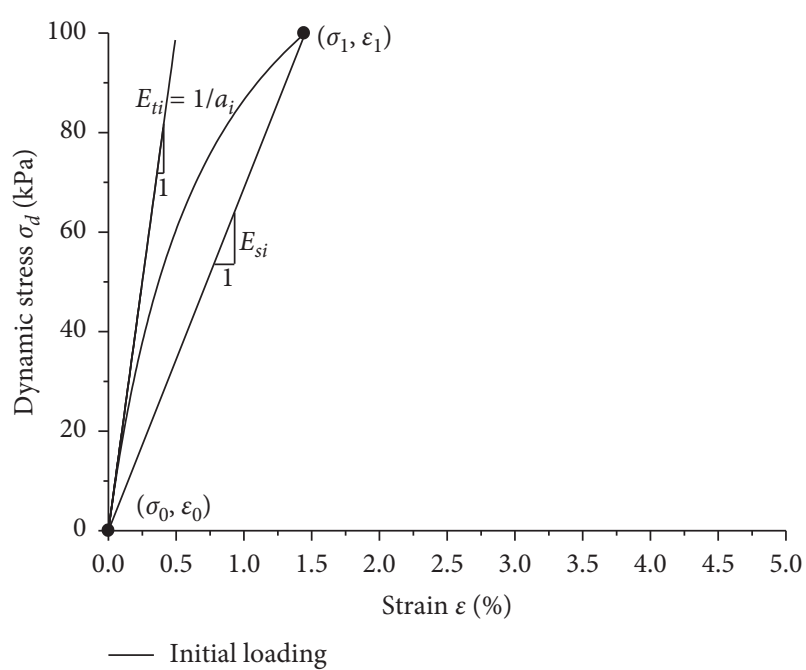

FIgURE 5: Significance of the parameter $a_{i}$ in the initial loading stage.

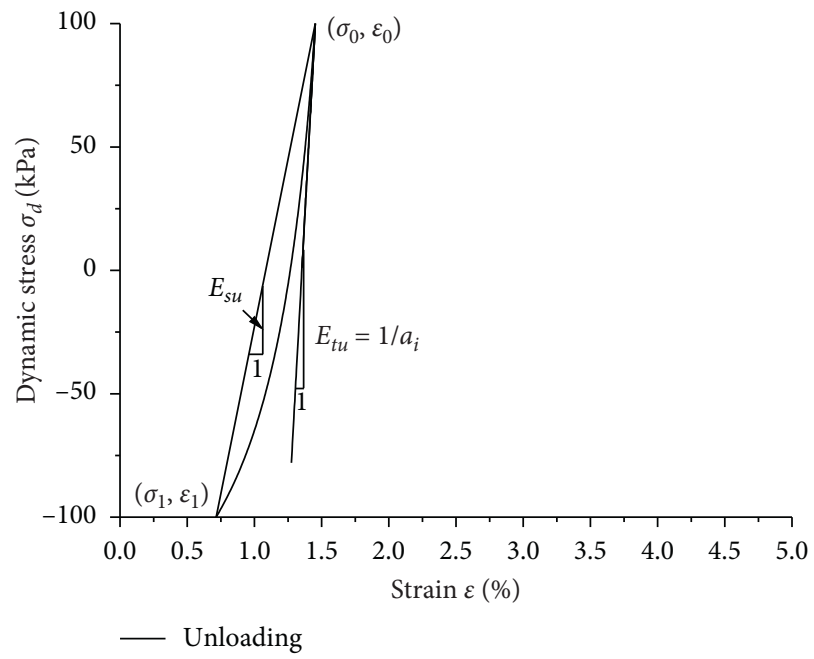

FIGURE 6: Significance of the parameter $a_{u}$ in the unloading stage.

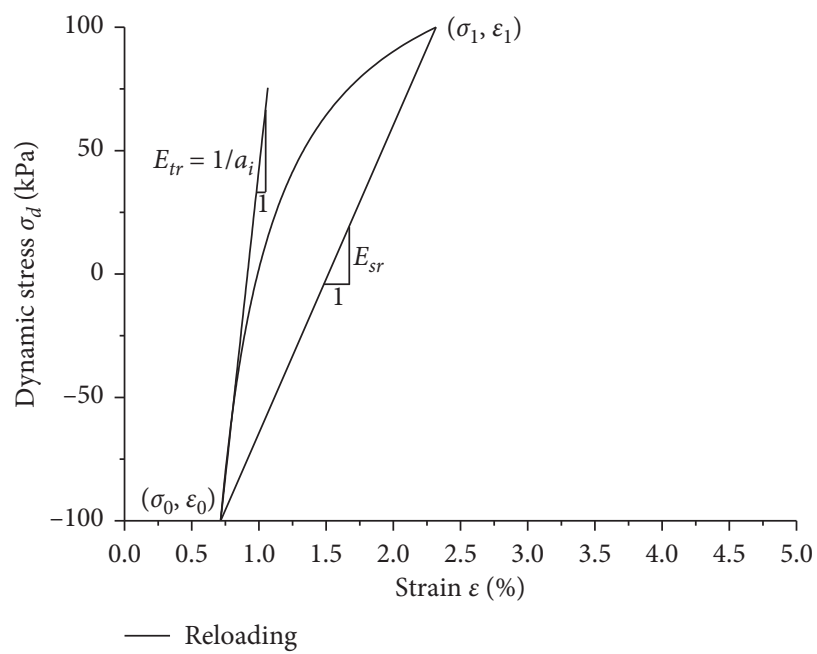

FIGURE 7: Significance of the parameter $a_{r}$ in the reloading stage. 
reloading) are $E_{s i}, E_{s u}$, and $E_{s r}$, respectively, and can be derived using the following formula:

$$
E_{s i}=E_{s u}=E_{s r}=\frac{\sigma_{1}-\sigma_{0}}{\varepsilon_{1}-\varepsilon_{0}} .
$$

3.2.1. Initial Loading Stage. In the initial loading stage, after loading from $\left(\sigma_{0}=0, \varepsilon_{0}=0\right)$ to $\left(\sigma_{1}, \varepsilon_{1}\right)$, equation (2) is converted as follows:

$$
\frac{\sigma_{1}}{\varepsilon_{1}}=\frac{1}{a_{i}+b_{i} \varepsilon_{1}}
$$

As

$$
\begin{aligned}
& \frac{\sigma_{1}}{\varepsilon_{1}}=\frac{\sigma_{1}-\sigma_{0}}{\varepsilon_{1}-\varepsilon_{0}}=E_{s i}, \\
& a_{i}=\frac{1}{E_{t i}},
\end{aligned}
$$

we have

$$
\frac{1}{E_{s i}}=\frac{1}{E_{t i}}+b_{i} \varepsilon_{1} .
$$

Using equation (9), we obtain

$$
\begin{array}{r}
\left(\frac{1}{E_{s i}}-\frac{1}{E_{t i}}\right) \frac{1}{\varepsilon_{1}}=b_{i} \\
\Rightarrow\left(\frac{1}{E_{s i}}-\frac{1}{E_{t i}}\right) \frac{\sigma_{1}}{\varepsilon_{1}} \frac{1}{\sigma_{1}}=b_{i} \\
\Rightarrow\left(\frac{1}{E_{s i}}-\frac{1}{E_{t i}}\right) E_{s i} \frac{1}{\sigma_{1}}=b_{i} \\
\Rightarrow\left(1-\frac{E_{s i}}{E_{t i}}\right) \frac{1}{\sigma_{1}}=b_{i} .
\end{array}
$$

It can be seen from Figure 5 and equation (10) that $E_{s i}<E_{t i}$, so $1-\left(E_{s i} / E_{t i}\right)>0$. As a result, $b_{i}>0$.

3.2.2. Unloading Stage. In the unloading stage, equation (3) can be used as the soil undergoes unloading from $\left(\sigma_{0}, \varepsilon_{0}\right)$ to $\left(\sigma_{1}, \varepsilon_{1}\right)$ (Figure 6); that is,

$$
\frac{\sigma_{1}-\sigma_{0}}{\varepsilon_{1}-\varepsilon_{0}}=E_{s u}=\frac{1}{a_{u}-b_{u}\left(\varepsilon_{1}-\varepsilon_{0}\right)} .
$$

Following the derivation method used for $b_{i}$ in the initial loading stage, we find

$$
b_{u}=\frac{1}{\varepsilon_{0}-\varepsilon_{1}}\left(\frac{1}{E_{s u}}-\frac{1}{E_{t u}}\right)=\frac{1}{\sigma_{0}-\sigma_{1}}\left(1-\frac{E_{s u}}{E_{t u}}\right) .
$$

Figure 1 illustrates that, during the cyclic loading test, the dynamic stress has a symmetrical amplitude $\sigma_{d}^{\text {amp }}$, namely, $\left|\sigma_{1}\right|=\sigma_{0}$. Thus,

$$
b_{u}=\frac{1}{2 \sigma_{0}}\left(1-\frac{E_{s u}}{E_{t u}}\right) \text {. }
$$

As $E_{s u}>0, E_{t u}<0$, and $\sigma_{0}>0$, it follows that $b_{u}>0$.

3.2.3. Reloading Stage. Similarly, in the reloading stage, after the soil is subjected to reloading from $\left(\sigma_{0}, \varepsilon_{0}\right)$ to $\left(\sigma_{1}, \varepsilon_{1}\right)$ (shown in Figure 7), the formula for $b_{r}$ is obtained:

$$
b_{r}=\frac{1}{\varepsilon_{1}-\varepsilon_{0}}\left(\frac{1}{E_{s r}}-\frac{1}{E_{t r}}\right)=\frac{1}{\sigma_{1}-\sigma_{0}}\left(1-\frac{E_{s r}}{E_{t r}}\right) .
$$

As $\sigma_{1}=\left|\sigma_{0}\right|$, this can be written as

$$
b_{r}=\frac{1}{2\left|\sigma_{0}\right|}\left(1-\frac{E_{s r}}{E_{t r}}\right) \text {. }
$$

3.3. Determination of the Model Parameters. From the description of the model parameters $a$ and $b$, it is noted that the model parameters can be obtained from the original test datum. Parameter $a$ is the initial tangent modulus of each loading or unloading stage, which means that it can be attained by using the ratio between $\sigma$ and $\varepsilon$ in the first very small increment of each stage; that is, $a_{\alpha}=(\Delta \sigma / \Delta \varepsilon)(\alpha=i, u$, or $r$ ).

As for parameter $b$, it can be calculated by using the initial tangent modulus $E_{t \alpha}$, the secant modulus $E_{s \alpha}$, and $\left|\sigma_{1}-\sigma_{0}\right|$, as in equations (10), (12), and (14) corresponding to different stages.

\section{Integration Algorithm for the Model}

4.1. Tangent Modulus and Integration Algorithm. The integration algorithm is the key to developing the constitutive model. The integration process can be summarized as subdividing the loading or unloading process (using some finite element or difference) into several substeps and assigning a strain increment to each load step. Next, the strain increments are calculated using the constitutive model. In contrast to elastoplastic theory, it is not necessary when using the hypoelasticity theory to judge whether the plastic modification is essential or not nor to ascertain numerous internal variables of the model via iteration. As long as the strain increments are small enough, the curve can be approximated based on the hypoelasticity theory through linear integration.

Thus, in the different loading stages, the most crucial step is to find the tangent modulus $E_{t}$ of each loading step. The tangent modulus of the stress-strain curve is obtained by differentiation of the hyperbolic curve. Equations (2)-(4) show that, in the different loading stages (initial, unloading, and reloading), the tangent moduli are given by the following:

(i) Initial loading stage:

$$
E_{t i}=\frac{\mathrm{d} \sigma}{\mathrm{d} \varepsilon}=\frac{a_{i}}{\left(a_{i}+b_{i} \varepsilon\right)^{2}} .
$$


(ii) Unloading stage:

$$
E_{t u}=\frac{\mathrm{d} \sigma}{\mathrm{d} \varepsilon}=\frac{a_{u}}{\left[a_{u}-b_{u}\left(\varepsilon-\varepsilon_{0}\right)\right]^{2}} .
$$

(iii) Reloading stage:

$$
E_{t r}=\frac{\mathrm{d} \sigma}{\mathrm{d} \varepsilon}=\frac{a_{r}}{\left[a_{r}-b_{r}\left(\varepsilon-\varepsilon_{0}\right)\right]^{2}} .
$$

After the tangent modulus has been found, the stress is deduced according to the following integration computation:

$$
\sigma^{\text {new }}=\sigma^{\text {old }}+\mathrm{d} \sigma=\sigma^{\text {old }}+E_{t \alpha} \mathrm{d} \varepsilon, \quad(\alpha=i, u, \text { or } r) .
$$

It is worth noting that in the different loading stages (initial, unloading, and reloading), the tangent moduli of the model are all positive. However, the strain increment, $d \varepsilon$, is positive in the initial loading and reloading stages, while it is negative in the unloading stage (so, the stress increases in the loading stages and declines in the unloading stages).

4.2. Judging Stress Reversal. In our algorithm, judging stress reversal is the key to finding the stress because its accuracy determines the precision of the algorithm. Evidently, stress reversal here refers to judging if the load state of the model is changing from one state to another, for example, from loading to unloading, or from unloading to loading. The purpose of judging stress reversal is to find the key state, so that the stress in the next step can be calculated using the different tangent moduli. The judgment process and corresponding algorithm for stress reversal are displayed in Figure 8.

4.3. Whole Integration Procedure. According to the integration algorithm and stress reversal judging method, the complete integration procedure for the proposed hypoelastic dynamic constitutive model, including those steps described above, can be summarized as follows.

What should be noted is that, like the Duncan-Chang static constitutive model, the dynamic stress in Table 1 is $\sigma_{1}-\sigma_{3}$, so the proposed model is a $3 \mathrm{D}$ model in the principal stress space that can be translated into other general stress spaces. It should also be noted that it mainly aims at cases involving $1 \mathrm{D}$ vibration (the most common condition); if $2 \mathrm{D}$ or $3 \mathrm{D}$ vibrations are analysed, the algorithm must be adjusted accordingly.

\section{Effects of Parameters $a$ and $b$ on the Stress- Strain Curve}

Whether or not the model can properly describe the stressstrain behaviour of the soil under cyclic loads depends on the model parameters used. Six model parameters are employed in this study $\left(a_{i}\right.$ and $b_{i}$ in the initial loading stage, $a_{u}$ and $b_{u}$ in the unloading stage, and $a_{r}$ and $b_{r}$ in the reloading stage). To appreciate the influences of parameters $a$ and $b$ of the hyperbolic equation on the stress-strain curve, the parameters $a_{r}$ and $b_{r}$ (reloading stage) are taken as examples for further explanation.

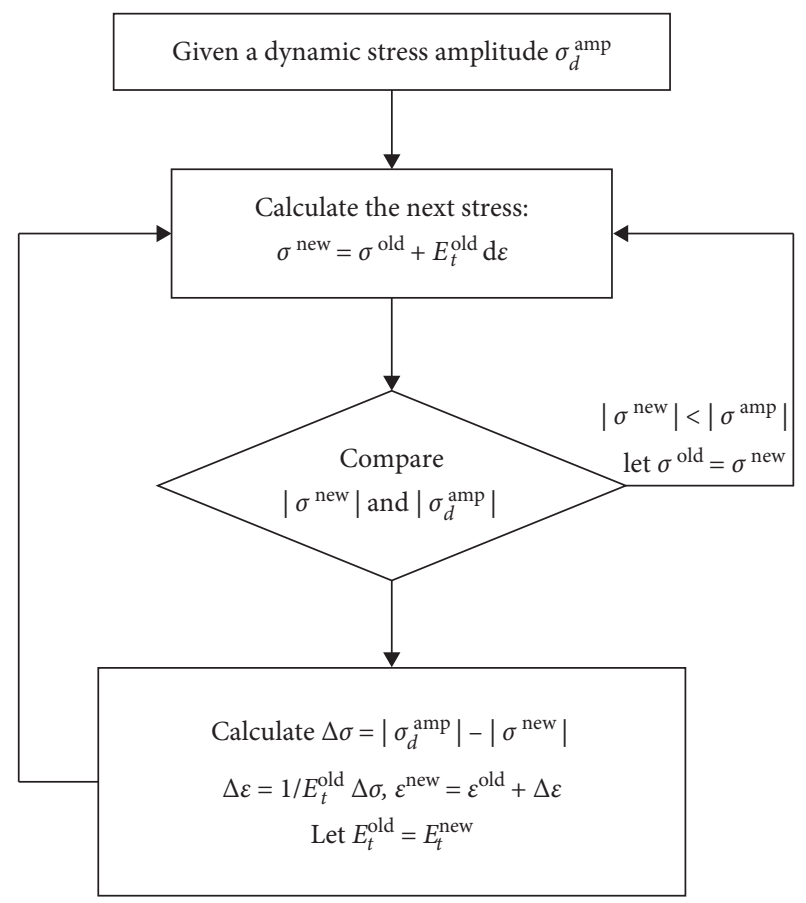

FIGURE 8: The procedure used in the integration scheme for stress reversal.

5.1. Effect of Parameter $a$ on the Stress-strain Curve. As mentioned above, parameter $a$ is related to the initial tangent modulus $E_{t}$ of the hyperbolic curve in terms of physical significance. Therefore, $a$ determines the inclination of the hyperbolic curve in the initial stage. Moreover, from a hysteresis perspective (Figure 9), parameter $a$ influences the damping ratio of the model. That is, the bigger the value of $a$, the larger the area bounded by the loading and unloading curve, so the larger the damping ratio. Conversely, a larger $a$ means a smaller damping ratio.

5.2. Effect of Parameter $b$ on the Stress-strain Curve. It can be seen from Figure 10 that parameter $b$ influences the curvature of the hyperbolic curve. The stress-strain curve is clearly more curved for larger values of parameter $b$. That is to say, in terms of physical significance, parameter $b$ directly affects the value and development rate of cumulative plastic strain. The smaller the value of $b$, the slower the development rate and the smaller the value of cumulative plastic strain (and vice versa).

Figure 11 demonstrates the effect of the combined action of parameters $a$ and $b$ on the stress-strain curve of the soil. It can be seen that when the same set of $a$ and $b$ values is used in the unloading and reloading stages $(a=1 / 80 \mathrm{E} 6, b=1$ / 3.0E5), the stress-strain curve forms a closed hysteresis loop (as the beginning and ending stresses in the two stages are superposed). However, the curves obtained from dynamic triaxial tests under cyclic loads show that this phenomenon does not occur in the initial loading and unloading stages. If we increase both $a$ and $b$ (to $a=1 / 60 \mathrm{E} 6, b=1 / 2.5 \mathrm{E} 5$ ), then the stress-strain curve starts to exhibit similar characteristics to those obtained in initial test curves. Thus, in the initial stage of a cyclic loading test, the soil is constantly compacted 
TABLE 1: The whole integration procedure.

\begin{tabular}{|c|c|}
\hline Step & Description \\
\hline 1 & Given a dynamic stress amplitude $\sigma_{d}^{\text {amp }}$, where $\sigma_{d}^{\text {amp }}=(1 / 2)\left(\sigma_{d}^{\max }-\sigma_{d}^{\min }\right)$ \\
\hline 2 & $\begin{array}{l}\text { Judge the loading or unloading stage according to the following condition: } \\
\text { (1) Initial loading stage: } \varepsilon_{0}=0, \mathrm{~d} \sigma=\sigma^{\text {new }}-\sigma^{\text {old }}>0 \\
\text { (2) Unloading stage: } \mathrm{d} \sigma=\sigma^{\text {new }}-\sigma^{\text {old }}<0 \\
\text { (3) Reloading stage: } \mathrm{d} \sigma=\sigma^{\text {new }}-\sigma^{\text {old }}>0\end{array}$ \\
\hline 3 & $\begin{array}{l}\text { Stress reversal judging stage: } \\
\text { If }\left|\sigma^{\text {new }}\right|>\left|\sigma_{d}^{\text {amp }}\right| \text {, then go to Step } 4 \\
\text { Else, } \sigma^{\text {old }}=\sigma^{\text {new }} \text { and go to Step } 5\end{array}$ \\
\hline 4 & $\begin{array}{l}\text { Calculate } \Delta \sigma=\left|\sigma_{d}^{\text {amp }}\right|-\left|\sigma^{\text {new }}\right| \\
\text { (1) } \Delta \varepsilon=\left(1 / E_{t \alpha}^{\text {old }}\right) \Delta \sigma \text {, where } \alpha \text { can be } i, u \text {, or } r \\
\text { (2) } \varepsilon^{\text {new }}=\varepsilon^{\text {old }}+\Delta \varepsilon, E_{t \alpha}^{\text {old }}=E_{t \alpha}^{\text {new }} \\
\text { (3) Record } \varepsilon_{0} \\
\text { Go to Step } 2\end{array}$ \\
\hline 5 & $\begin{array}{l}\text { Calculate the new strain } \varepsilon^{\text {new }} \text { : } \\
\text { (i) Initial loading stage: } \\
\text { (1) Firstly, calculate } E_{t i}=(\mathrm{d} \sigma / \mathrm{d} \varepsilon)=\left(a_{i} /\left(a_{i}+b_{i} \varepsilon^{\text {old }}\right)^{2}\right) \\
\text { (2) Then, } \Delta \varepsilon^{\text {new }}=\left(\Delta \sigma / E_{t i}\right), \Delta \sigma \text { is the trial increment of the strain and given automatically by the program } \\
\text { (ii) Unloading stage: } \\
\text { (1) Firstly, calculate } E_{t u}=(\mathrm{d} \sigma / \mathrm{d} \varepsilon)=\left(a_{u} /\left(a_{u}+b_{u} \varepsilon_{u}^{\text {old }}-\varepsilon_{0}\right)^{2}\right) \\
\text { (2) Then, } \Delta \varepsilon^{\text {new }}=\left(\Delta \sigma / E_{t u}\right) \\
\text { (iii) Reloading stage: } \\
\text { (1) Firstly, calculate } E_{t r}=(\mathrm{d} \sigma / \mathrm{d} \varepsilon)=\left(a_{r} /\left(a_{r}+b_{r} \varepsilon_{t r}^{\text {old }}-\varepsilon_{0}\right)^{2}\right) \\
\text { (2) Then, } \Delta \varepsilon^{\text {new }}=\left(\Delta \sigma / E_{t r}\right) \\
\text { Let } \varepsilon^{\text {new }}=\varepsilon^{\text {old }}+\Delta \varepsilon^{\text {new }}, E_{t \alpha}^{\text {old }}=E_{t \alpha}^{\text {new }} \text {, where } \alpha \text { can be } i, u \text {, or } r \\
\text { Go to Step } 2\end{array}$ \\
\hline
\end{tabular}

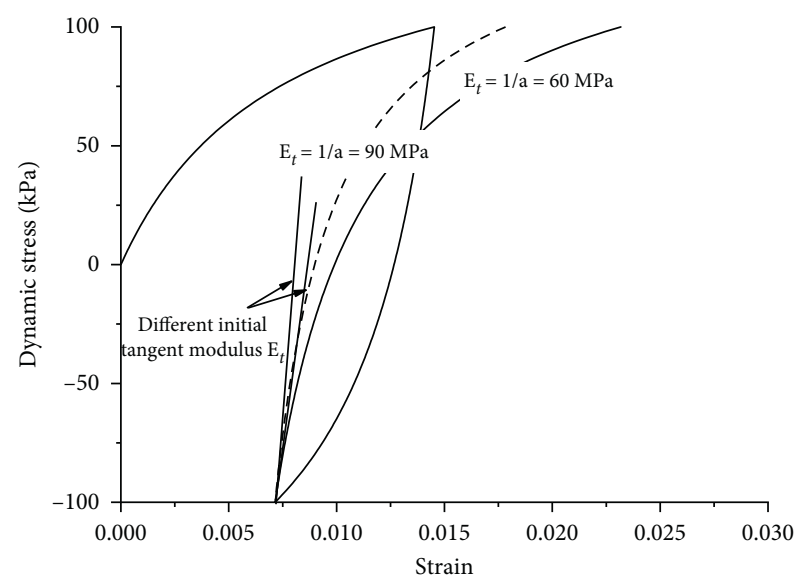

FIGURE 9: The effect of parameter a on the stress-strain curve.

under the cyclic load and so plastic strain develops cumulatively. Therefore, the values of $a$ and $b$ increase correspondingly. During the later stages of the test, the cumulative plastic strain of the soil will stop developing. Hence, the hysteresis loops formed in the stress-strain curve will become closed and the values of $a$ and $b$ will tend to remain the same.

\section{Applications}

To verify the rationality of the proposed model, it is used to simulate the stress-strain data obtained from dynamic triaxial tests under conventional triaxial and cyclic loads.

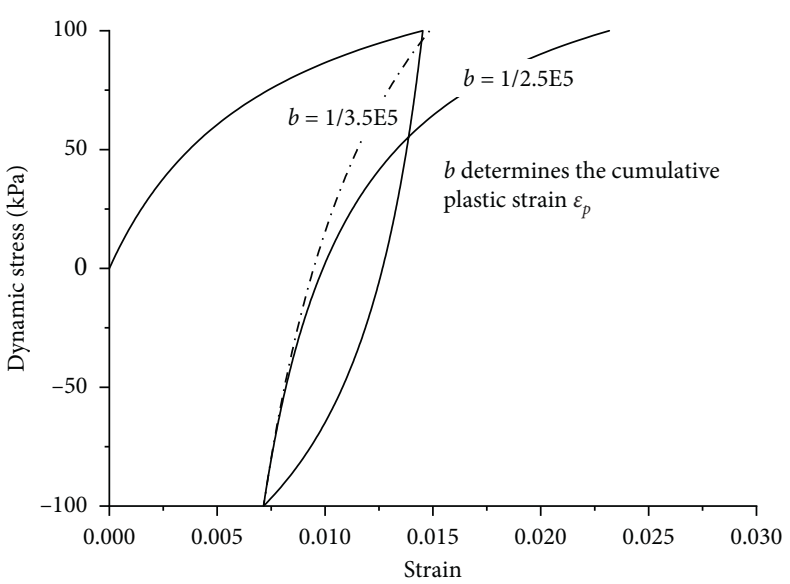

Figure 10: Effect of parameter b on the stress-strain curve.

6.1. Conventional Triaxial Tests. If loading occurs monotonically, then stress reversal will not occur. This implies that the model merely experiences the initial loading stage. Therefore, the model is reduced to the commonly seen Duncan-Chang model. However, it is worth noting that because of the model's algorithm, a large dynamic stress amplitude $\sigma_{\mathrm{amp}} d$ needs to be specified before simulating the conventional triaxial test results (as stress reversal occurs when $\sigma>\sigma_{\text {amp }} d$ ).

Conventional triaxial tests were performed using red clay (compactness value of 0.8 ) as the test soil. Experiments, employing confining pressures of 50,100 , and $200 \mathrm{kPa}$ were conducted. Figure 12 displays the results of the conventional 


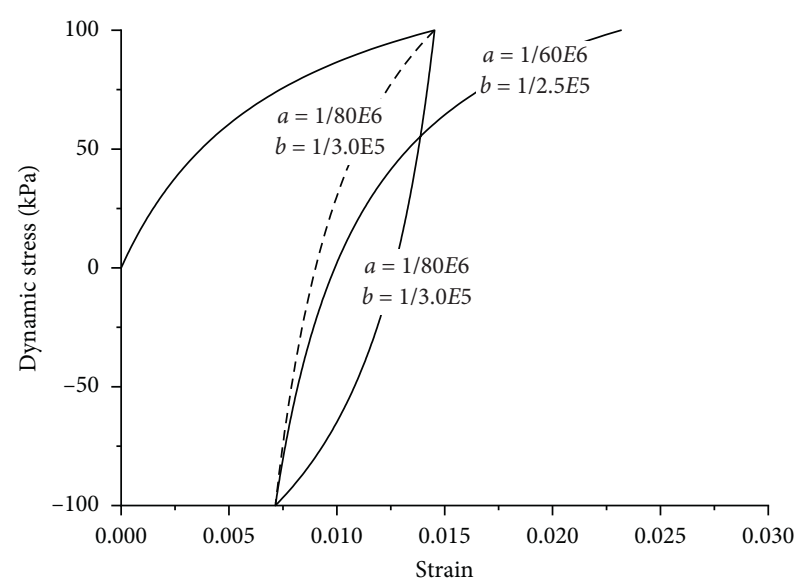

FIGURE 11: The combined effect of parameters $a$ and $b$ on the stressstrain curve.

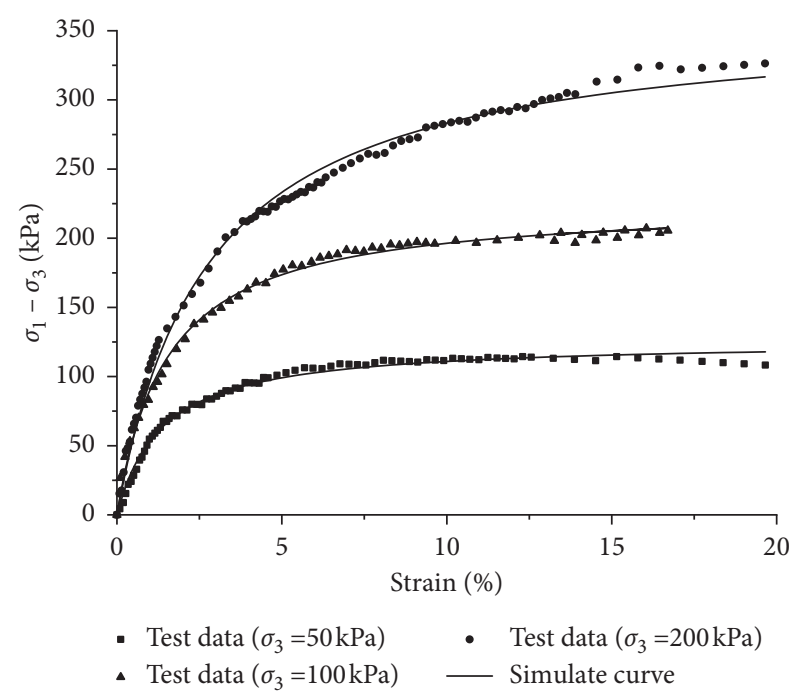

FIgURE 12: Simulation of conventional triaxial tests.

triaxial tests and simulation results. In the simulations, the values of the $a$ and $b$ parameters used in the constitutive model are 1/16.7E6 and 1/1.26E2; 1/32.6E6 and 1/2.26E2; and $1 / 60.1 \mathrm{E} 6$ and $1 / 3.61 \mathrm{E} 2$, respectively, for increasingly high pressures. It can be seen that the simulated values calculated using the model are in good agreement with the experimental results.

6.2. Dynamic Triaxial Tests under Cyclic Loads. To validate the model under cyclic loading conditions, the results obtained by Huang et al. [31] were simulated in this section. In the tests reported, standard sand was used so that the dynamic elastic modulus and damping ratio of the samples under cyclic loading could be investigated without draining. The test conditions were set as follows: the dry density of the sand was $1.58 \mathrm{~g} / \mathrm{cm}^{3}$, the confining pressure was $300 \mathrm{kPa}$, and the consolidation ratio was 1.5 .

Figure 13 shows the experimental curves obtained and simulation results. It can be seen that the stress-strain curves of the soil under the conditions used are well described by the

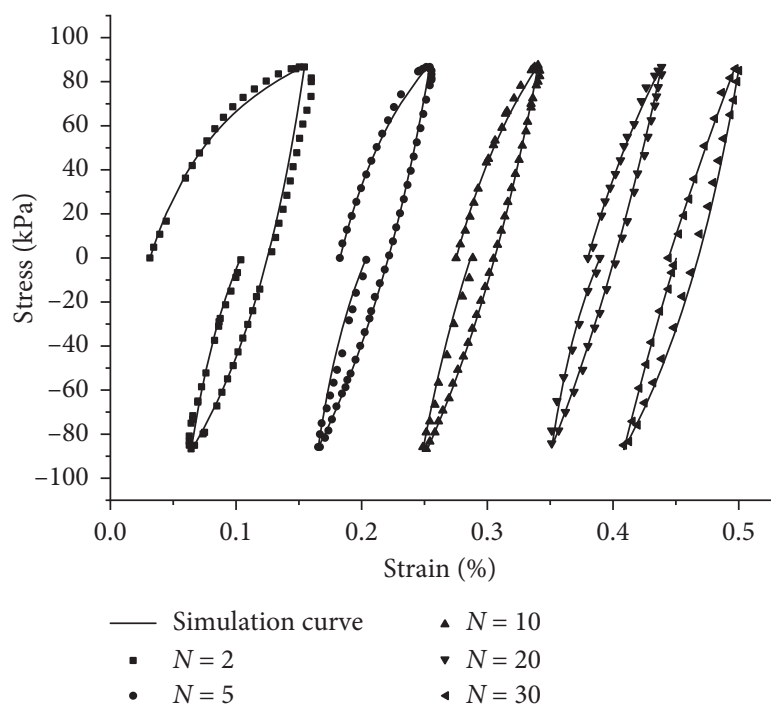

FIgURE 13: Test data and simulation curves corresponding to a cyclic load.

model constructed in the present study. In the initial stages of the test, the stress-strain hysteresis loops are clearly not closed owing to the occurrence of residual deformation. However, the residual deformation gradually stabilizes and the hysteresis loops tend to become closed after a few cycles. This is consistent with the conclusions of Hardin et al. [32]. (These authors suggested that when the consolidation ratio is 1.0, the hysteresis loops tend to be closed. When it is larger than 1.0, the hysteresis loops are not closed at first due to the existence of initial shear stress. Subsequent hysteresis loops, however, can be considered to be closed after a certain number of cycles.)

Figure 14 illustrates typical nonclosed hysteresis loops and also highlights certain quantities that may be used to define the dynamic elastic modulus. Consider the "peak" and "valley" points (A and B) of the particular loop shown. Then, the dynamic stress $\sigma_{d}$ and dynamic strain $\varepsilon_{d}$ are determined as follows:

$$
\begin{gathered}
\sigma_{d}=\frac{\sigma_{d A}-\sigma_{d B}}{2}, \\
\varepsilon_{d}=\frac{\varepsilon_{d A}-\varepsilon_{d B}}{2} .
\end{gathered}
$$

The dynamic elastic modulus $\left(E_{d}\right)$ and damping ratio $\left(\lambda_{d}\right)$ are given by

$$
\begin{aligned}
& E_{d}=\frac{\sigma_{d}}{\varepsilon_{d}} \\
& \lambda_{d}=\frac{2}{\pi} \frac{A_{\text {shadow }}}{A_{T}},
\end{aligned}
$$

where $A_{\text {shadow }}$ represents half of the area in the hysteresis loop while $A_{T}$ denotes the area of the triangle ABC.

Figures 15 and 16 illustrate how the dynamic elastic modulus and damping ratio change for the test soil according to the data. The graphs also show the values obtained from the simulations. The agreement between the simulated and experimental results is shown to be very good. 


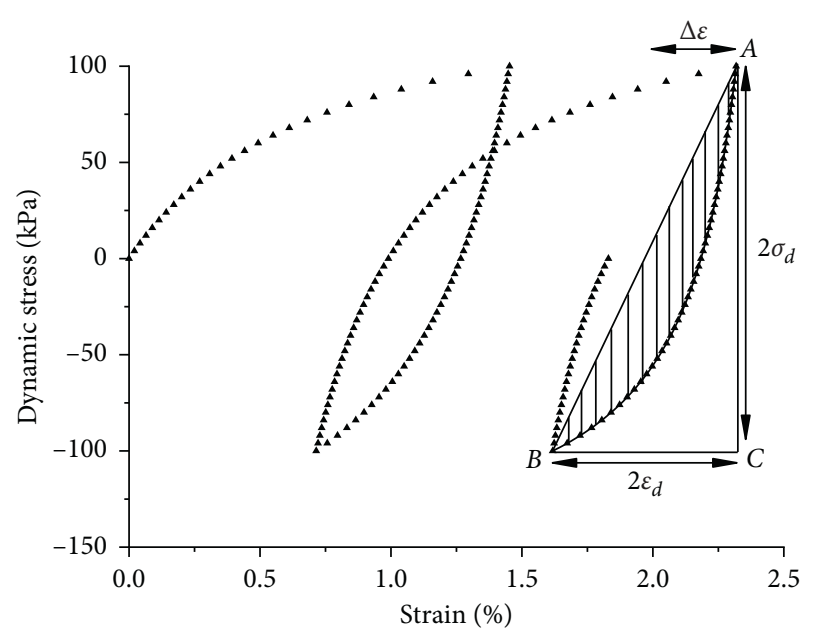

FIgURE 14: Nonclosed stress-strain hysteresis loops.

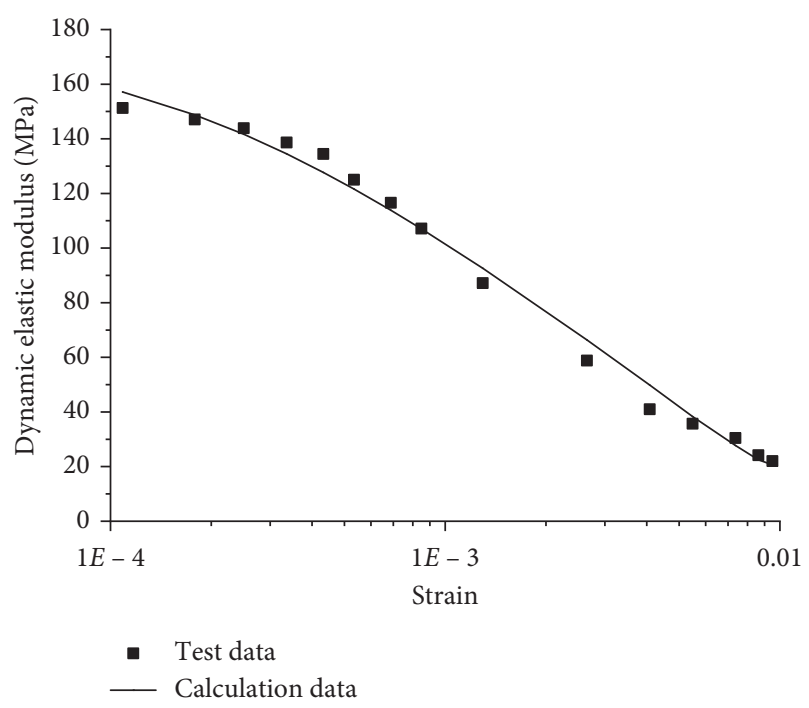

FIgURE 15: The relationship between $E_{d}$ and $\varepsilon_{d}$.

A closer inspection of Figures 13 and 15 shows that the hysteresis loop of the soil in the initial stage of cyclic loading is more obviously inclined and there is a larger value of dynamic strain compared to the loops towards the end of the cyclic loading process. Thus, the dynamic elastic modulus is smaller. Moreover, the hysteresis loops become closed and their inclinations gradually decrease as the number of cycles increases. Meanwhile, the value of the dynamic strain declines, and so the dynamic elastic modulus increases.

A closer inspection of Figures 13 and 16 shows that the damping ratio is directly related to the hysteresis loop occurring in the soil. In the initial stage, the soil exhibits an obvious hysteretic behaviour and the hysteresis loops have a larger inclination. Therefore, the damping ratio is small. However, towards the end of the loading process, the hysteresis loops become gradually closed while the hysteretic behaviour and inclination decline. Thus, the damping ratio increases.

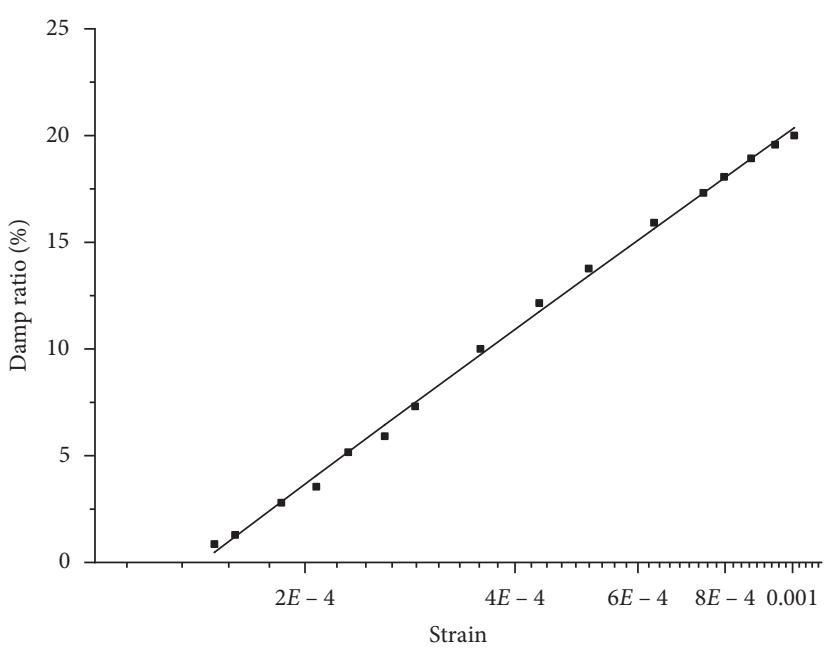

- Test data

- Calculation data

Figure 16: The relationship between $\lambda_{d}$ and $\varepsilon_{d}$.

\section{Conclusions}

Using the hypoelasticity theory and simple hyperbolic equations, a novel dynamic constitutive model was established and applied. The model does not require complex elastoplastic judgments to be made and so has a simple theoretical form which is convenient to develop and apply. We firstly provided the sources of inspiration used to construct the model, as a result of which the stress in the soil was divided into three loading stages. Hyperbolic functions were then established for the stress-strain relationships associated with each loading stage. The physical significance and method for the determination of the model parameters were then discussed and the effects of the parameters on the stress-strain behaviour of the soil are demonstrated. An integration algorithm for the model and a method of recognizing dynamic stress reversal were then proposed, which make the development of the model highly convenient. Finally, the effectiveness of the model constructed in the study was confirmed by comparison with results from conventional and cyclic loading triaxial tests. The results show that the stress-strain behaviour of the soil can be well described by the model.

\section{Data Availability}

No data were used to support this study.

\section{Conflicts of Interest}

The authors declare that there are no conflicts of interest regarding the publication of this paper.

\section{Acknowledgments}

This research was funded by the National Natural Science Foundation of China (Grant nos. 41702337 and 51709258). 


\section{References}

[1] A. M. Kaynia, C. Madshus, and P. Zackrisson, "Ground vibration from high-speed trains: prediction and countermeasure," Journal of Geotechnical and Geoenvironmental Engineering, vol. 126, no. 6, pp. 531-537, 2000.

[2] J. Wang, Study on Dynamic Behavior of Saturated Soft Clay under Unidirectional and Bidirectional Cyclic Loading, Zhejiang University, Hangzhou, China, 2007.

[3] Q. Yang, M. Wang, and M. T. Luan, "Experimental research of correlation on static and dynamic strength of unsaturated silty clay," Rock and Soil Mechanics, vol. 31, no. 1, pp. 71-80, 2010.

[4] H. B. Wei, H. B. Liu, and Y. P. Gao, "Experimental research on dynamic properties of fly ash soil subjected to freeze-thaw cycles," Rock and Soil Mechanics, vol. 28, no. 5, pp. 1005-1008, 2007.

[5] C. Hu, H. Liu, and W. Huang, "Anisotropic bounding-surface plasticity model for the cyclic shakedown and degradation of saturated clay," Computers and Geotechnics, vol. 44, pp. 3447, 2012

[6] Y. Xiao, Y. Sun, H. Liu, J. Xiang, Q. Ma, and L. Long, "Model predictions for behaviors of sand-nonplastic-fines mixturesusing equivalent-skeleton void-ratio state index," Science China Technological Sciences, vol. 60, no. 6, pp. 878-892, 2017.

[7] L.-Y. Xu, J.-Z. Zhang, F. Cai, W.-Y. Chen, and Y.-Y. Xue, "Constitutive modeling the undrained behaviors of sands with non-plastic fines under monotonic and cyclic loading," Soil Dynamics and Earthquake Engineering, vol. 123, pp. 413-424, 2019.

[8] Z. Mróz, V. A. Norris, and O. C. Zienkiewicz, “An anisotropic hardening model for soils and its application to cyclic loading," International Journal for Numerical and Analytical Methods in Geomechanics, vol. 2, pp. 203-221, 1978.

[9] Z. Mróz, V. A. Norris, and O. C. Zienkiewicz, "Application of an anisotropic hardening model in the analysis of elastoplastic deformation of soils," Géotechnique, vol. 29, no. 1, pp. 1-34, 1979.

[10] J. H. Prevest, "Mathematical modeling of monotonic and cyclic undrained clay behaviour," International Journal for Numerical and Analytical Methods in Geomechanics, vol. 1, pp. 195-216, 1977.

[11] N. Khutia, P. P. Dey, S. K. Paul, and S. Tarafder, "Development of non Masing characteristic model for LCF and ratcheting fatigue simulation of SA333 C-Mn steel," Mechanics of Materials, vol. 65, pp. 88-102, 2013.

[12] L. S. Tang, H. R. Liao, Z. X. Liu, and Q. H. Zhang, "Advances in research on mechanical behavior of the subgrade soils under dynamic load," Geological Science and Technology Information, vol. 25, no. 2, pp. 103-112, 2006.

[13] Z. H. Yang and J. H. Wang, "Anisotropic bounding surface model for saturated soft clay under cyclic loading," Rock and Soil Mechanics, vol. 37, no. S1, pp. 63-71, 2016.

[14] X. L. Chen, J. H. Wang, and S. Z. Li, "Elastoplastic simulation for undrained cyclic stress-strain responses of soft clays," Chinese Journal of Geotechnical Engineering, vol. 36, no. 05, pp. 933-941, 2014.

[15] C. Hu, H. X. Liu, and W. Huang, "Damage-dependent bounding surface model for cyclic degradation of saturated clay," Rock and Soil Mechanics, vol. 33, no. 2, pp. 459-466, 2012.

[16] X. G. Fang and Y. C. Xiong, "A multi-surface model considering shear and compression damages," Chinese Journal of Rock Mechanics and Engineering, vol. S2, pp. 3501-3506, 2006.
[17] H. Y. Zhuang, G. X. Chen, and D. H. Zhu, "Dynamic viscoplastic memorial nested yield surface model of soil and its verification," Chinese Journal of Geotechnical Engineering, vol. 10, pp. 1267-1272, 2006.

[18] Y. F. Dafalias, M. T. Manzari, and M. Akaishi, "A simple anisotropic clay plasticity model," Mechanics Research Communications, vol. 29, no. 4, pp. 241-245, 2002.

[19] Y. F. Dafalias and M. Taiebat, "SANISAND-Z: zero elastic range sand plasticity model," Géotechnique, vol. 66, no. 12, pp. 999-1013, 2016.

[20] Y. F. Dafalias, "Must critical state theory be revisited to include fabric effects?" Acta Geotechnica, vol. 11, no. 3, pp. 479-491, 2016.

[21] Y. F. Dafalias and M. Taiebat, "Rotational hardening with and without anisotropic fabric at critical state," Géotechnique, vol. 64 , no. 6, pp. 507-511, 2014.

[22] Y. F. Dafalias and M. Taiebat, "Anatomy of rotational hardening in clay plasticity," Géotechnique, vol. 63, no. 16, pp. 1406-1418, 2013.

[23] D. Y. Xie, Z. H. Wu, and Y. T. Guo, "Application of limit equilibrium theory to the analysis of dynamic failure process of saturated sand," China Civil Engineering Journal, vol. 14, no. 4, pp. 17-28, 1981.

[24] D. Y. Xie, Z. H. Wu, and Y. T. Guo, "The application of critical equilibrium theory to the dynamic failure process of saturated sand," Journal of Northwest A\&F University (Natural Science Edition), vol. 1, pp. 1-14, 1981.

[25] D. Y. Xie and J. M. Zhang, "Developing mechanism on transient strength deformation of saturated sand under cyclic loading," China Civil Engineering Journal, vol. 20, no. 3, pp. 57-70, 1987.

[26] Z. P. Bazant and A. Yavari, "Is the cause of size effect on structural strength fractal or energetic-statistical?" Engineering Fracture Mechanics, vol. 72, no. 1, pp. 1-31, 2005.

[27] Z. P. Bazant and P. D. Bhat, "Endochronic theory of inelasticity and failure of concrete," ASCE Journal of Engineering Mechanics Division, vol. 102, no. EM4, pp. 701-722, 1976.

[28] Z. P. Bazant, A. M. Ansal, and R. J. Krizek, "Viscoplasticity of transversely isotropic clay," ASCE Journal Engineering Mechanics Division, vol. 105, no. 4, pp. 549-565, 1979.

[29] O. C. Zienkiewicz, C. T. Chang, and E. Hinton, "Non-linear seismic response and liquefaction," Internal Journal for $\mathrm{Nu}$ merical and Analytical Methods in Geomechanics, vol. 20, no. 5, pp. 307-329, 1978.

[30] J. M. Duncan and C. Y. Chang, "Nonlinear analysis of stress and strain in soil," Journal of the Soil Mechanics and Foundations Division, vol. 96, no. 5, pp. 1629-1633, 1970.

[31] B. Huang, X. D. Fu, and B. J. Zhang, "Test technology and normalized characteristics of dynamic elastic modulus and damping ratio," Chinese Journal of Geotechnical Engineering, vol. 37, no. 4, pp. 659-666, 2015.

[32] B. O. Hardin and V. P. Drnevich, "Shear modulus and damping in soils: measurement and parameter effects (terzaghi leture)," Journal of the Soil Mechanics and Foundations Division, vol. 98, no. 6, pp. 603-624, 1972. 